\title{
Involvement of minor components associated with the FimA fimbriae of Porphyromonas gingivalis in adhesive functions
}

\section{Correspondence \\ So-ichiro Nishiyama \\ nisiyama@dpc.agu.ac.jp}

Received 24 December 2006

Revised 15 February 2007

Accepted 19 February 2007

\author{
So-ichiro Nishiyama, ${ }^{1}$ Yukitaka Murakami, ${ }^{1}$ Hideki Nagata, ${ }^{2}$ \\ Satoshi Shizukuishi, ${ }^{2}$ Ikuro Kawagishi ${ }^{3}$ and Fuminobu Yoshimura ${ }^{1}$
}

\author{
${ }^{1}$ Department of Microbiology, School of Dentistry, Aichi-Gakuin University, 1-100 Kusumoto-cho, \\ Chikusa-ku, Nagoya, Aichi 464-8650, Japan \\ ${ }^{2}$ Department of Preventive Dentistry, Graduate School of Dentistry, Osaka University, \\ 1-8 Yamadaoka, Suita, Osaka 565-0871, Japan \\ ${ }^{3}$ Department of Biological Science, Graduate School of Science and Institute for Advanced \\ Research, Nagoya University, Chikusa-ku, Nagoya 464-8602, Japan
}

\begin{abstract}
The FimA fimbriae of Porphyromonas gingivalis, the causative agent of periodontitis, have been implicated in various aspects of pathogenicity, such as colonization, adhesion and aggregation. In this study, the four open reading frames (ORF1, ORF2, ORF3 and ORF4) downstream of the fimbrilin gene (fimA) in strain ATCC 33277 were examined. ORF2, ORF3 and ORF4 were demonstrated to encode minor components of the fimbriae and were therefore renamed fimC, fimD and fimE, respectively. Immunoblotting analyses revealed that inactivation of either fim $C$ or fimD by an ermF-ermAM insertion, but not inactivation of ORF1, was accompanied by concomitant loss of the products from the downstream genes, raising the possibility that fimC, fimD and fimE constitute a transcription unit. The fimE mutant produced FimC and FimD, but fimbriae purified from it contained neither protein, suggesting that FimE is required for the assembly of FimC and FimD onto the fimbrilin (FimA) fibre. The fim C, fimD and fimE mutants lost autoaggregation abilities. Fimbriae purified from these three mutants showed attenuated binding activities to glyceraldehyde-3-phosphate dehydrogenase of Streptococcus oralis and to two extracellular matrix proteins, fibronectin and type I collagen. These results suggest that FimE, as well as FimC and FimD, play critical roles in the adhesive activities of the mature FimA fimbriae in $P$. gingivalis.
\end{abstract}

\section{INTRODUCTION}

Porphyromonas gingivalis is a Gram-negative, black-pigmented obligate anaerobe that has been implicated in adult periodontitis (Lamont \& Jenkinson, 1998), which is a major cause of tooth loss in the adult population. This bacterium settles in the gingival crevice by forming a mixed-species consortium with a number of other Gramnegative and -positive bacteria. $P$. gingivalis can also invade and persist within gingival epithelial cells (Andrian et al., 2006). Consistent with these modes of colonization, the adhesion of the bacterium to host tissues and other oral bacteria via its fimbriae has been implicated in its pathogenicity.

Abbreviations: $\mathrm{CBB}$, Coomassie brilliant blue; GAPDH, glyceraldehyde3-phosphate dehydrogenase; rGAPDH, recombinant glyceraldehyde-3phosphate dehydrogenase; Sup, supernatant; WC, whole cultures.

The GenBank/EMBL/DDBJ accession no. for the fimA, ORF1, fimC, fimD, and fimE sequence of $P$. gingivalis is D42067.
$P$. gingivalis has at least two distinct types of fimbriae (Lamont \& Jenkinson, 2000) composed of distinct fimbrilins, FimA (the fimA gene product) and Mfal (the $m f a 1$ gene product), with apparent molecular masses of about 38 and $75 \mathrm{kDa}$, respectively. Both types of fimbriae appear to be abundant in some strains and represent evolutionarily unique groups, since no homologues of FimA or Mfa1 have been found in any other bacteria. The FimA fimbriae are approximately $0.3-1.6 \mu \mathrm{m}$ in length and $\sim 5 \mathrm{~nm}$ in diameter (Yoshimura et al., 1984), whereas the Mfa1 $(75 \mathrm{kDa})$ fimbriae are slightly thicker $(3.5-6.5 \mathrm{~nm}$ in width) and considerably shorter (80-120 nm in length) than the FimA fimbriae (Park et al., 2005).

FimA fimbriae are known to bind to a number of eukaryotic proteins such as fibronectin, collagen, laminin (Hamada et al., 1998), saliva-derived proline-rich protein (Amano et al., 1999) and statherin (Amano et al., 1996), as well as to a prokaryotic protein, glyceraldehyde-3-phosphate dehydrogenase (GAPDH) of Streptococcus oralis 
(Maeda et al., 2004b). Interactions between recombinant FimA ( $\mathrm{rFimA}$ ) and some of these proteins were demonstrated with the biomolecular interaction analysis system (BIAcore) (Kontani et al., 1996; Maeda et al., 2004a).

The fimA genes from various $P$. gingivalis strains have been classified into six types (I, Ib, II, III, IV and V) (Amano et al., 2004), and each strain carries a single fimA gene of one of these types (Dickinson et al., 1988; Takahashi et al., 1992). It has been reported that a large majority of periodontitis patients carry organisms with either type II or IV fimA, while type I is the most prevalent fimA genotype among $P$. gingivalis-positive healthy adults, although no morphological differences in the fimbriae were reported (Amano et al., 2000). Fimbriae of type I strains (381 and ATCC 33277) have been extensively studied since the 1980s, although the complete genome sequence of this type has not been reported. The genome sequence of the poorly fimbriated strain W83 (type IV) has been completed (Nelson et al., 2003).

In many bacteria, the gene encoding the major fimbrilin typically forms a cluster with several additional genes encoding regulatory factors, fimbrial minor components or transport machineries (Soto \& Hultgren, 1999). P. gingivalis also has a set of fimbriae-related genes flanking the fimA gene. Nishikawa et al. (2004) identified fimX, a gene upstream of fimA, as a target of the FimS/FimR twocomponent regulatory system which controls the expression of fimA. Watanabe et al. (1996) sequenced the region $(\sim 5 \mathrm{~kb})$ downstream of fimA and identified four open reading frames (ORF1, ORF2, ORF3 and ORF4). The ORF2 and ORF3 products were reported to be minor components in fimbriae purified from strain 381 and inferred to play critical roles in fimbrial functions (Yoshimura et al., 1993). Takahashi et al. (2001) expressed the fimA gene of strain 381 in various $P$. gingivalis strains and found that the transformants showed reduced ability to bind some bacteria and mammalian epithelial cells, as well as reduced autoaggregation activity. These results imply that some components that are present on the native fimbriae but absent on the recombinant fimbriae are, at least partly, involved in the adhesion function of FimA fimbriae. Recently, Hajishengallis et al. (2006) reported that the wild-type fimbriae can utilize a Toll-like receptor (TLR), TLR1 or TLR6, for cooperative TLR2-dependent activation of transfected cell lines, but the mutant fimbriae lacking the minor components displayed a preference for TLR1 and a stronger inflammatory response than the wildtype fimbriae, suggesting that fimbriae with or without the minor components are discriminated by the innate immune system.

During the course of isolation of fimbria-defective mutants by transposon mutagenesis and purification of fimbriae from ATCC 33277 and its mutant derivatives, we found that ORF4 was longer than previously reported and its size was closely comparable to that of a previously unidentified minor component $(60 \mathrm{kDa})$ of fimbriae purified from strain 381. Here we report the characterization of the genes downstream of fimA and their products, by sequencing the region of two genotype I strains and by constructing isogenic mutants of ATCC 33277 with insertion of an erythromycin-resistance cassette in each gene. The products of three genes, ORF2, ORF3 and ORF4, were found to be minor components of wild-type fimbriae and therefore the genes were renamed fim , fimD and fimE, respectively (the new nomenclature will be used hereafter). Their products were also shown to play critical roles in autoaggregation and binding to several bacterial and eukaryotic proteins.

\section{METHODS}

Bacterial strains and growth conditions. All $P$. gingivalis and Escherichia coli strains used in this study are listed in Table 1 . The $P$. gingivalis strain ATCC 33277 and its derivatives were grown on blood agar plates (Brucella HK agar base, Kyokuto) supplemented with $5 \%$ (w/v) laked rabbit blood, $2.5 \mu \mathrm{g}$ haemin $\mathrm{ml}^{-1}, 5.0 \mu \mathrm{g}$ menadione $\mathrm{ml}^{-1}$ and $0.01 \%(\mathrm{w} / \mathrm{v})$ DTT at $37{ }^{\circ} \mathrm{C}$ under anaerobic conditions. Cells were then cultivated in sTSB liquid medium [trypticase soy broth supplemented with $0.25 \%(\mathrm{w} / \mathrm{v})$ yeast extract, $2.5 \mu \mathrm{g}$ haemin $\mathrm{ml}^{-1}, 5.0 \mu \mathrm{g}$ menadione $\mathrm{ml}^{-1}$ and $\left.0.01 \%(\mathrm{w} / \mathrm{v}) \mathrm{DTT}\right]$ at $37{ }^{\circ} \mathrm{C}$ for 48-60 h under anaerobic conditions. For secondary cultivation, the primary culture was inoculated into sTSB medium at a $1: 100$ dilution and incubated at $37^{\circ} \mathrm{C}$ for an additional $24 \mathrm{~h}$. The cells were then harvested and used for subsequent analyses. When necessary, gentamicin (Gm; $200 \mu \mathrm{g} \mathrm{ml}^{-1}$ ) or erythromycin (Em;20 $\mathrm{g} \mathrm{ml}^{-1}$ ) was added to each medium. E. coli strains were grown in LB medium supplemented, when necessary, with kanamycin $\left(50 \mu \mathrm{g} \mathrm{ml}{ }^{-1}\right)$ or erythromycin $\left(200 \mu \mathrm{g} \mathrm{ml}^{-1}\right)$.

Cloning and sequencing of ORF1, fimC (formerly ORF2), fimD (formerly ORF3) and fimE (formerly ORF4) in P. gingivalis strains 381 and ATCC 33277. Strain SN47-631, isolated as a transposon-generated mutant defective in fimbriation, had an insertion in fimE (formerly ORF4), raising the possibility that the gene is involved in biogenesis and function of the FimA fimbriae. We therefore screened a plasmid library of chromosomal DNA from strain 381 (Hasegawa et al., 2003) for fimE-positive clones by using primers NSN220 and NSN164 (see Table 2 for primer sequences). Sequencing was carried out using the ABI PRISM Version 1.1 kit and ABI PRISM 3100-Avant Genetic Analyser (Applied Biosystems). The revised nucleotide sequence data were deposited in DDBJ (accession no. D42067). Based on the sequence information of the fimEencompassing region from strain 381, a primer set (NSN172 and 227) was designed to amplify the corresponding region from the chromosomal DNA of ATCC 33277. The $2.0 \mathrm{~kb}$ fragment was amplified by PCR and cloned into the plasmid vector pCR-BluntITOPO (from the Zero Blunt TOPO PCR cloning kit, Invitrogen). Also, the 1.0, 1.4 and $2.1 \mathrm{~kb}$ fragments spanning from ORF1 to fimC (formerly ORF2), from fim $C$ to fimD (formerly ORF3) and from fimD to fimE, respectively, were amplified and cloned into the TOPO vector. The entire DNA sequence from ORF1 to fimE of ATCC 33277 was identical to that of strain 381 . To construct a plasmid expressing recombinant FimE protein (rFimE), the fimE region encoding the putative mature product (nucleotides 112 to 1650) was amplified by PCR using NSN242 and NSN243 as primers and the chromosomal DNA of ATCC 33277 as the template. The resulting fragment was cloned into the expression vector pET200/D-TOPO (Champion pET directional TOPO expression kit, Invitrogen), yielding plasmid pSN140 encoding rFimE His-tagged at its N-terminus. The plasmid 
Table 1. Strains used in this study

\begin{tabular}{|c|c|c|}
\hline Strain & Relevant characteristics ${ }^{\star}$ & Source or reference \\
\hline \multicolumn{3}{|c|}{ Porphyromonas gingivalis } \\
\hline 381 & Heavily fimbriated strain & Yoshimura et al. (1984) \\
\hline ATCC 33277 & Type strain from ATCC, heavily fimbriated & Laboratory stock \\
\hline SN47-631 & Mutant carrying a transposon insertion in fimE, $\mathrm{Em}^{\mathrm{r}}$ & This study \\
\hline KDP98 & fimA insertion mutant of ATCC 33277, $\mathrm{Em}^{\mathrm{r}}$ & Watanabe-Kato et al. (1998) \\
\hline KO1 & ORF1 deletion mutant of ATCC $33277, \mathrm{Em}^{\mathrm{r}}$ & This study \\
\hline OZ5001C & ORF2 (fimC) insertion mutant of ATCC 33277, $\mathrm{Em}^{\mathrm{r}}$ & Hongo et al. (1999) \\
\hline $\mathrm{KO} 3$ & ORF3 (fimD) insertion mutant of ATCC $33277, \mathrm{Em}^{\mathrm{r}}$ & This study \\
\hline $\mathrm{KO} 4$ & ORF4 (fimE) insertion mutant of ATCC 33277, $\mathrm{Em}^{\mathrm{r}}$ & This study \\
\hline \multicolumn{3}{|l|}{ Escherichia coli } \\
\hline TOP 10 & $\begin{array}{c}\mathrm{F}^{-} \text {mcrA } \Delta(\text { mrr-hsdRMS-mcrBC) } \phi 80 \text { lacZ } \Delta \mathrm{M} 15 \Delta \text { lacX74 recA1 } \\
\text { araD139 } \Delta(\text { ara-leu }) 7697 \text { galU galK rpsL }\left(\text { Str }^{\mathrm{r}}\right) \text { endA1 nupG }\end{array}$ & Invitrogen \\
\hline BL21 star (DE3) & $\mathrm{F}^{-}$ompT hsd $S_{\mathrm{B}}\left(r_{B}^{-} m_{B}^{-}\right) \mathrm{gal} d c m$ rne131 (DE3) & Invitrogen \\
\hline $\mathrm{DH} 5 \alpha$ & $\begin{array}{l}\mathrm{F}^{-} \text {d80lacZ } \triangle \mathrm{M} 15 \Delta(\text { lacZYA-argF }) \text { U169 deoR recA1 endA1 } \\
\text { hsdR17 }\left(r_{K}^{-} m_{K}^{+}\right) \text {phoA supE44 } \lambda^{-} \text {thi-1 gyrA96 relA1 }\end{array}$ & Takara Bio \\
\hline
\end{tabular}

${ }^{\star} \mathrm{Em}^{\mathrm{r}}$, resistance to erythromycin.

was introduced into E. coli strain BL21 star (DE3) for overproduction and purification of rFimE.

Protein purification and preparation of antibody. Purification of FimA fimbriae from $P$. gingivalis was performed by a method described previously (Yoshimura et al., 1984) with slight modifications. Purification of $\mathrm{rFimE}$ or recombinant glyceraldehyde-3phosphate dehydrogenase (rGAPDH) (Maeda et al., 2004a) was carried out with a Ni-NTA purification system (Invitrogen), following the manufacturer's instructions. Protein concentrations were measured by using the Micro BCA protein assay kit (Pierce Biotechnology). Polyclonal antibody against rFimE was raised in rabbits as described previously (Yoshimura et al., 1984).

Insertion mutagenesis. All mutants used in this study were derivatives of ATCC 33277 resulting from an erythromycin resistance

Table 2. Primers used in this study

\begin{tabular}{|ll|}
\hline Name & \multicolumn{1}{c|}{ Sequence $\left(\mathbf{5}^{\prime} \mathbf{- 3}^{\prime}\right)^{*}$} \\
\hline NSN164 & CTTCCCGGAGCTTGGCTTTC \\
NSN172 & ATGACGGGCAGGTATTATTGG \\
NSN201 & GTAGTCCCGAAGGATGATGG \\
NSN206 & CTGCAGGTCGACTCTAGAG \\
NSN220 & CAAGCTCTACGACGCCAGC \\
NSN227 & CCTGAAAGCGTGGCGCGAG \\
NSN229 & CACTCGTAAATGCAGACGC \\
NSN235 & GCTATCGGGGGTACCAACAATGATTCTTTGATTG \\
NSN236 & CTAGAGTCGACCTGCAGTTCAGGCTTTCCTCTAC- \\
& CG \\
NSN242 & CACCGTAAGCGGTAGCATTGTTTC \\
NSN243 & CTAATTCGTCACGTGAATCG \\
\hline
\end{tabular}

${ }^{\star}$ Underlining indicates the overlapping regions of the $5^{\prime}$ or $3^{\prime}$ end of ermF-ermAM. gene $\left(\mathrm{Em}^{\mathrm{r}}\right)$ cassette (ermF-ermAM) replacing or being inserted into the gene of interest (Fletcher et al., 1995). The ORF1 mutant (KO1) was constructed by the primer extension method as described previously (Nagano et al., 2005). In brief, the entire coding region of ORF1 (Fig. 1, indicated by a double-headed arrow) in ATCC 33277 was replaced with ermF-ermAM by PCR using primers NSN201, 206, 229, 235 and 236 (Table 2). Gene replacement in the resulting strain was confirmed by PCR. The fimC mutant (OZ5001C), which has an $\mathrm{Em}^{\mathrm{r}}$ cassette insertion at the SnaBI site (745 bp) in the fimC coding region, was described previously (Hongo et al., 1999). The fimD mutant (KO3), which carries an insertion of the whole plasmid sequence including the $\mathrm{Em}^{\mathrm{r}}$ cassette between the HincII and BamHI sites in the fimD coding region, was constructed as follows. The HincII-BamHI fragment ( $335 \mathrm{bp}$ ) of fimD was cloned into the suicide vector pVAL7 (Smith \& Salyers, 1989). The resulting plasmid, pVAL7-80, was introduced into ATCC 33277 via conjugation and $\mathrm{Gm}^{\mathrm{r}} \mathrm{Em}^{\mathrm{r}}$ transconjugants were selected. The fimE mutant (KO4), which carries an insertion of the $\mathrm{Em}^{\mathrm{r}}$ cassette at the Eco81I site ( $384 \mathrm{bp}$ ) in the fimE coding region, was made by a similar method to that used for the construction of the $g p p X$ mutant of $P$. gingivalis (Hasegawa et al., 2003).

Sample preparation for detection of fimbrilin and fimbrial minor components. FimA and the products of the genes downstream of fimA expressed from $P$. gingivalis cells were detected after precipitation with TCA as described previously (Hongo et al., 1999) except that $2 \mathrm{M}$ Tris was used to neutralize samples instead of washing with diethyl ether. Almost all the bacterial components, including minor, protease-sensitive proteins, could be quantitatively recovered by the TCA precipitation method because strong intrinsic proteolytic activities possessed by this organism were instantaneously inactivated (Hongo et al., 1999). For the preparation of samples from 'whole cultures' (WC), $100 \mu 1100 \%$ (w/v) TCA (Nacalai Tesque) was added directly to $900 \mu \mathrm{l}$ of each cell culture. Each culture was also fractionated by centrifugation $(14000 \mathrm{~g}, 3 \mathrm{~min}$ ). Cells collected from $900 \mu \mathrm{l}$ of each culture were resuspended in $1 \mathrm{ml} 10 \%$ (w/v) TCA ('Cell'), whereas $100 \mu 1100 \%(\mathrm{w} / \mathrm{v}$ ) TCA was added to the culture supernatant ('Sup'). In all cases, samples were then incubated on ice for $15 \mathrm{~min}$ and subjected to centrifugation $(20000 \mathrm{~g}, 20 \mathrm{~min})$. After the supernatant was removed, $10 \mu 12 \mathrm{M}$ Tris was added to the pellet, 


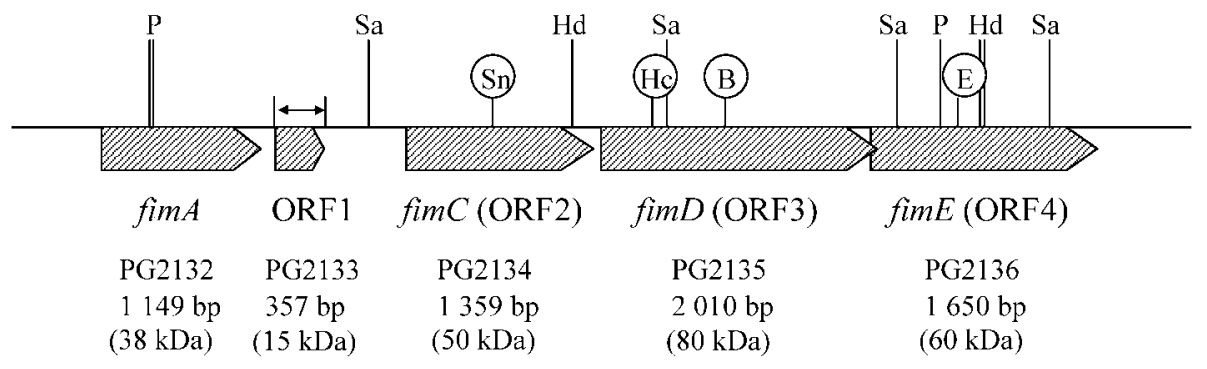

Fig. 1. Schematic diagram of the fim gene cluster in $P$. gingivalis strain ATCC 33277. The fim $A$ and other coding regions are represented by hatched bars with pointed ends indicating their $3^{\prime}$ ends. The genes downstream of fim $A$ have been referred to as ORF1, ORF2, ORF3 and ORF4 in the literature (Watanabe et al., 1996); the latter three were renamed fimC, fimD and fimE, respectively, in this study since they were shown to encode minor components of the FimA fimbriae. The PG numbers (from PG2132 to PG2136) below the bars indicate the orthologous ORFs in P. gingivalis W83, which were annotated by TIGR. The length of the genes (bp) and the deduced molecular masses of the products are also shown below the bars. Major restriction sites are indicated, with those sites used to insert the $\mathrm{Em}^{\mathrm{r}}$ cassette to construct the fim $\mathrm{C}$, fimD and fimE mutants circled (B, BamHI; E, Eco81l; Hc, Hincll; Hd, Hindlll; P, Pstl; Sa, Sacl; Sn, SnaBI). The double-headed arrow indicates the region exchanged with the $\mathrm{Em}^{\mathrm{r}}$ cassette to construct the ORF1 mutant (see Methods for details).

which was then resuspended in distilled water and SDS loading buffer. The final sample volume was adjusted according to the $\mathrm{OD}_{600}$ values measured before harvest.

SDS-PAGE and immunoblotting. SDS-PAGE and immunoblotting were carried out as described previously (Lugtenberg et al., 1975; Yoshimura et al., 1989). Proteins were separated with a $12 \%(\mathrm{w} / \mathrm{v})$ SDS-PAGE gel and visualized by staining with Coomassie brilliant blue (CBB) R-250. For immunoblotting, proteins were transferred onto a nitrocellulose membrane (Hybond ECL nitrocellulose membrane, GE Healthcare Biosciences) and detected by using antibody raised against fimbrilin (FimA monomer) (Yoshimura et al., 1984), ORF1, FimC, FimD (Watanabe et al., 1996), or FimE (this study), and peroxidase-conjugated goat anti-rabbit IgG (MP Biomedicals). Signals were visualized with $0.01 \%(w / v)$ 4-chloro-1naphthol in $20 \mathrm{mM}$ Tris/ $\mathrm{HCl}(\mathrm{pH}$ 7.5) containing $0.5 \mathrm{M} \mathrm{NaCl}$ supplemented with hydrogen peroxide. To increase sensitivity for the detection of certain proteins, ECL Plus Western blotting detection reagents (GE Healthcare Biosciences) were used for visualization according to the manufacturer's instructions. Before immuoblotting, samples of the same group were analysed by SDS-PAGE, followed by dye-staining. Then, their protein patterns and the intensities of stained protein bands were carefully compared with each other to verify that they had an equivalent amount of proteins for quantitative immunoblotting analysis.

Autoaggregation assay. Cells were grown as described above, harvested by centrifugation at $8000 \mathrm{~g}$ for $10 \mathrm{~min}$, gently washed twice with $20 \mathrm{mM}$ PBS, pH 6.0, and resuspended in $20 \mathrm{mM}$ PBS. PBS adjusted to $\mathrm{pH} 6.0$ was used throughout the autoaggregation assays. The $\mathrm{OD}_{550}$ value of the cell suspension was measured and adjusted by dilution with PBS to 1.0 , which corresponds to $1.0 \times 10^{8} \mathrm{cells} \mathrm{ml}^{-1}$ (Amano et al., 1997). Aliquots (2 $\mathrm{ml}$ each) in test tubes $(13 \mathrm{~mm}$ diameter) were then shaken at room temperature at a speed of 120 strokes $\min ^{-1}$. At $0,5,10,15,20,30$ and $40 \mathrm{~min}$, the $\mathrm{OD}_{550}$ values of the suspensions were measured with a spectrophotometer (mini photo 518R; TAITEC).

Binding assay. Binding of fimbriae to prokaryotic and eukaryotic proteins was examined by ELISA using polystyrene microtitre plates (96-well, Maxisorp; Nalge Nunc) or type I collagen pre-coated plates (Becton Dickinson Labware). Coating of the former with purified
rGAPDH $\left(100 \mu \mathrm{l}\right.$ of 10 or $20 \mu \mathrm{g} \mathrm{ml}^{-1}$ in $50 \mathrm{mM} \mathrm{Na} \mathrm{CO}_{3} / \mathrm{NaHCO}_{3}$ buffer, $\mathrm{pH}$ 9.5) and with fibronectin (Sigma-Aldrich; $100 \mu \mathrm{l}$ of 10 or $20 \mu \mathrm{g} \mathrm{ml}^{-1}$ dissolved in $20 \mathrm{mM} \mathrm{PBS}, \mathrm{pH} 7.2$ ) were carried out at $4{ }^{\circ} \mathrm{C}$ overnight and at $37{ }^{\circ} \mathrm{C}$ for $2 \mathrm{~h}$, respectively (for fibronectin coating see Nakamura et al., 1999). PBS adjusted to $\mathrm{pH} 7.2$ was used throughout the binding assays. As a negative control, buffer-only wells $\left(100 \mu \mathrm{l} 50 \mathrm{mM} \mathrm{Na} \mathrm{CO}_{3} / \mathrm{NaHCO}_{3}\right.$ buffer or $\left.100 \mu \mathrm{l} \mathrm{PBS}\right)$ were prepared and subjected to the same treatments. After washing three times with PBS containing $0.05 \%$ (w/v) Tween-20, the wells were incubated with $300 \mu \mathrm{l} 4 \%$ (w/v) casein (Block Ace; Snow Brand) at room temperature for $2 \mathrm{~h}$ for blocking, washed again, and incubated with $100 \mu$ purified fimbriae ( 2.5 or $5.0 \mu \mathrm{g} \mathrm{ml}^{-1}$ in PBS) at room temperature for $2 \mathrm{~h}$ and then at $4{ }^{\circ} \mathrm{C}$ overnight. After washing, the wells were treated with $100 \mu \mathrm{l} 4 \%$ (w/v) casein or PBS containing anti-fimbriae (polymer) antibody (Yoshimura et al., 1984) (at a $1: 8000$ dilution) and peroxidase-conjugated goat anti-rabbit IgG (at a $1: 16000$ dilution) (MP Biomedicals). Then the EIA peroxidase substrate, 3,3',5,5'-tetramethylbenzidine (Bio-Rad; $100 \mu \mathrm{l}$ ), was added to each well. The reactions were stopped by adding $100 \mu \mathrm{l}$ $0.5 \mathrm{M} \mathrm{H}_{2} \mathrm{SO}_{4}$. The binding activities were assessed by measuring the $A_{450}$ values with a microplate reader (model 680 ; Bio-Rad). All assays were carried out in triplicate and the standard errors were determined. Experiments with pre-coated collagen plates were performed similarly except that coating and blocking procedures were skipped. As a negative control, PBS $(100 \mu \mathrm{l})$ without fimbriae protein was applied to several wells which were then subjected to the same procedures as those with fimbriae. For each combination, the mean $A_{450}$ value of the control reactions was subtracted from that of the triplicate assays with fimbriae and the resulting value was defined as net binding.

\section{RESULTS}

\section{Genes downstream of fimA in type I strains}

In this study, we focused on the four ORFs (ORF1, fimC, fimD and fimE) downstream of the fimA gene encoding fimbrilin in $P$. gingivalis (Fig. 1). The sequences of the four ORFs in strain 381 have already been published (Watanabe et al., 1996). Comparing the fimA gene clusters between 
strains W83 (Nelson et al., 2003) and 381, we found that ORF1 and fimE in 381 are shorter than the corresponding genes (PG2133 and PG2136, respectively) of W83. We sequenced this region in both 381 and ATCC 33277 and found that the size of the fimE coding region of 381 (1650 bp) is identical to that of ATCC 33277 and very similar to that of the PG2136 coding region of W83 (1662 bp). The ORF1 sequence of strain 381 was also identical to that of ATCC 33277, but was shorter than that of PG2133 in W83. A schematic diagram of the region downstream of fimA in ATCC 33277, used hereafter as the parent strain, is presented in Fig. 1.

\section{The fimE gene encodes a minor component of the FimA fimbriae}

The fimC and fimD products co-purified with FimA fimbriae and therefore appeared to be minor components of the fimbriae (Yoshimura et al., 1993), whereas the ORF1 and fimE products were not detected in the same preparation (Watanabe et al., 1996). Here we raised a polyclonal antibody against full-length recombinant FimE, and examined fimbriae purified from ATCC 33277 by immunoblotting with the new antibody. FimE was detected in the purified fimbriae from the wild-type strain (Fig. 2, lane WT in panel Anti-FimE). The apparent molecular mass of the product was approximately $60 \times 10^{3}$, in good agreement with that deduced from the nucleotide sequence (60639.43 for the precursor form and 56672.91 for the predicted mature form). FimE, as well as FimC and FimD, could be detected by CBB staining when sufficient amounts (more than $30 \mu \mathrm{g}$ protein) of the wild-type fimbriae were applied (data not shown). These results demonstrate that FimE is produced in $P$. gingivalis and is associated with FimA fimbriae, and therefore we conclude that FimE, like FimC and FimD, is a minor component of the FimA fimbriae. On the other hand, the ORF1 product was not detected in the fimbriae preparation (data not shown) as reported previously (Watanabe et al., 1996).

\section{Effects of mutations of ORF1, fimC, fimD and fimE on the levels of the minor components and fimbrilin}

We constructed and characterized a series of mutants with the $\mathrm{Em}^{\mathrm{r}}$ cassette (ermF-ermAM) inserted into ORF1, fimC, fimD or fimE. First, whole cultures (WC) of the wild-type and mutant strains, as well as supernatants (Sup) and pellets (Cell) after low-speed centrifugation of the whole cultures, were analysed by immunoblotting with antibodies raised against ORF1, FimC, FimD and FimE after precipitating proteins with TCA to minimize degradation by endogenous proteases of $P$. gingivalis, which is a potent proteolytic organism (Hongo et al., 1999). The ORF1 product was not detected in any sample tested (data not shown). The ORF1 mutant had slightly lower amounts of FimC, FimD and FimE than the wild-type strain (Fig. 3, compare lanes ORF1 and WT in panels FimC/WC through
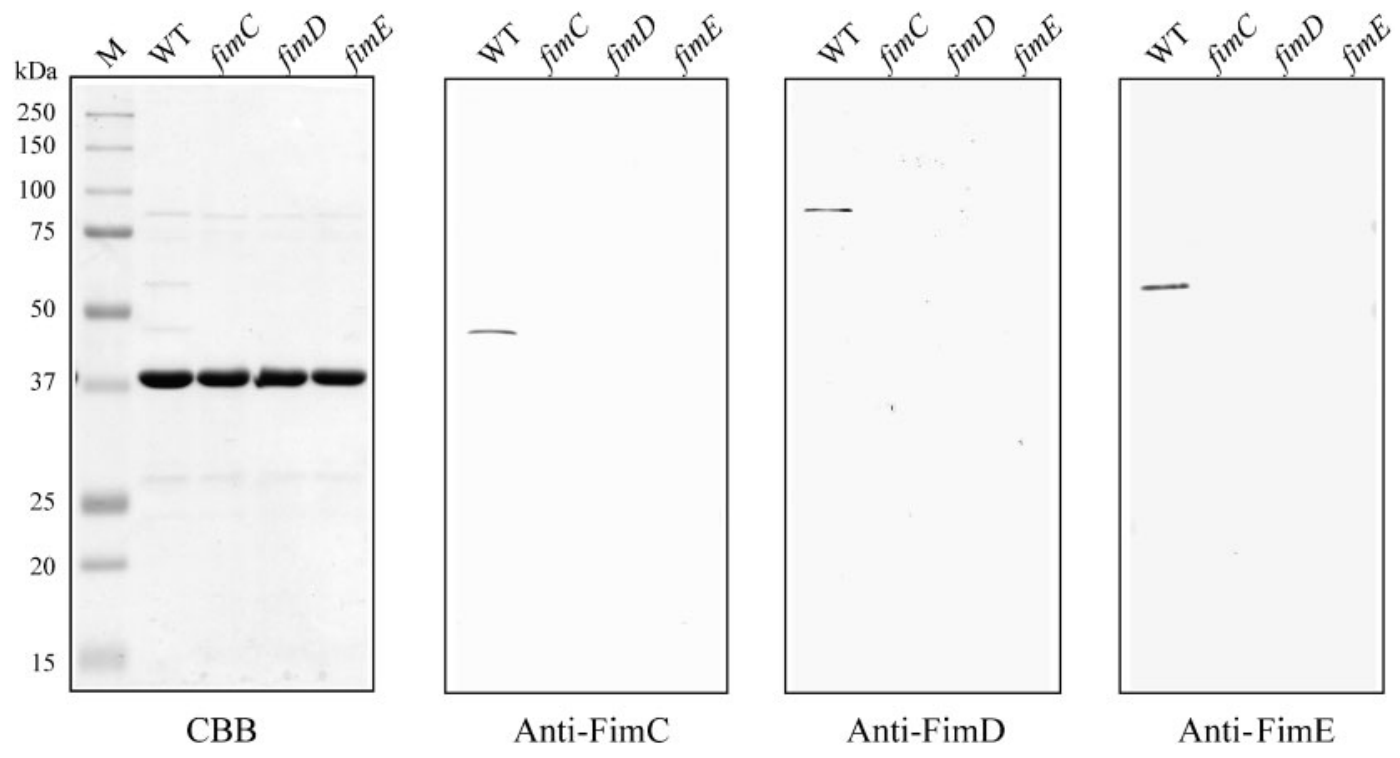

Fig. 2. Detection of fimbrilin (FimA) and minor components in purified fimbriae from the wild-type and mutant $P$. gingivalis strains. Fimbriae $(10 \mu \mathrm{g}$ each) purified from the wild-type strain (ATCC 33277) and its fimC and fimE derivatives were subjected to SDS-PAGE followed by Coomassie brilliant blue staining (CBB) and immunoblotting with antibodies raised against recombinant FimC (Anti-FimC), FimD (Anti-FimD) and FimE (Anti-FimE). Lanes: M, markers; WT, wild-type strain ATCC 33277; fim C, fim C mutant OZ5001C; fimD, fimD mutant KO3fimE, fimE mutant KO4. 

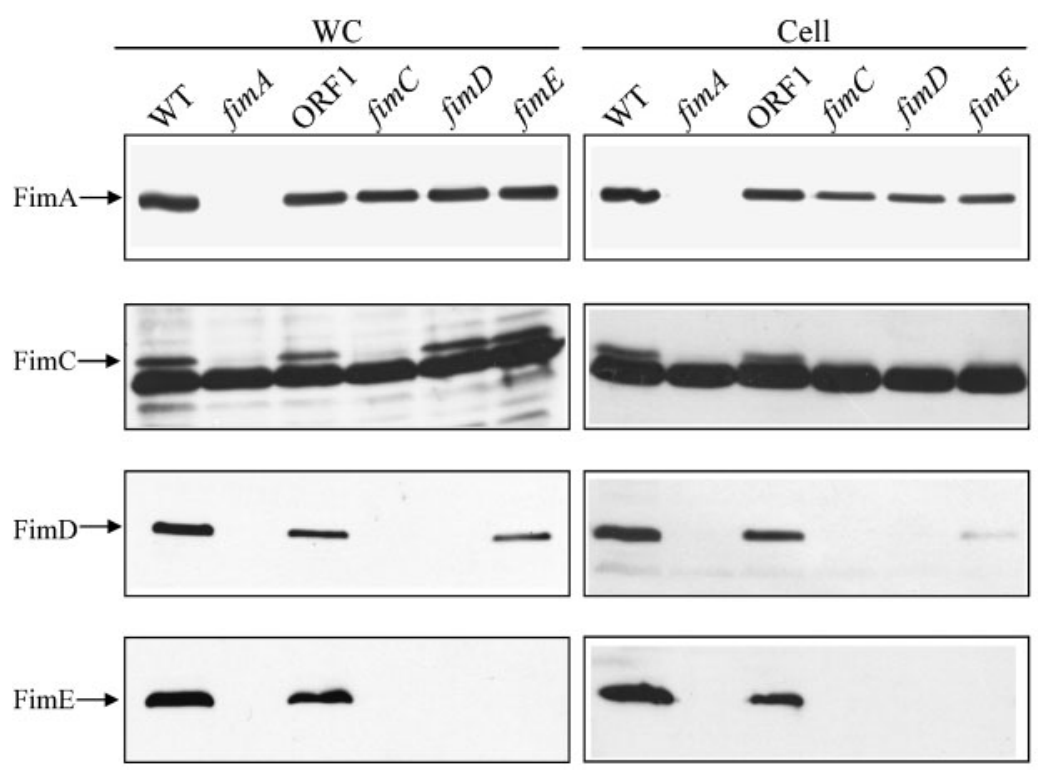
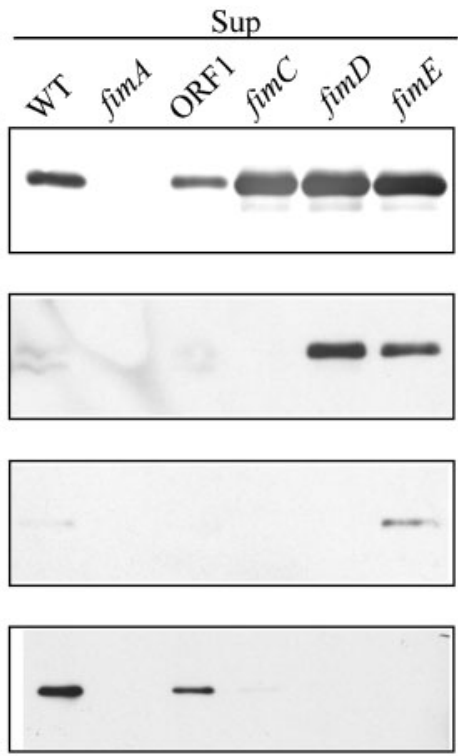

Fig. 3. Detection of fimbrilin (FimA) and minor components in cultures of the wild-type and mutant $P$. gingivalis strains. Proteins from whole cultures (panels labelled WC) of the wild-type and mutant strains, as well as those from their pellets (panels labelled Cell) and supernatants (panels labelled Sup) after low-speed centrifugation (14000 g, $3 \mathrm{~min}$ ), were precipitated with TCA and subjected to SDS-PAGE followed by immunoblotting with antibodies raised against fimbrilin (FimA monomer) and recombinant minor components (bands indicated with FimA, FimC, FimD and FimE). To detect signals of FimC, FimD and FimE, the ECL Plus system (GE Healthcare Biosciences) was used. Arrows indicate corresponding protein bands. Each lane contained a sample corresponding to $50 \mu \mathrm{l}$ (FimA panels labelled WC and Cell), $200 \mu \mathrm{l}$ (all panels labelled Sup), or $100 \mu \mathrm{l}$ (all other panels) of cell culture with an $\mathrm{OD}_{600}$ value of 1.0. Lanes: WT, wild-type strain ATCC 33277; fimA, fimA mutant KDP98; ORF1, ORF1 mutant $\mathrm{KO}$; fimC, fimC mutant OZ5001C; fimD, fimD mutant KO3; fimE, fimE mutant KO4.

FimE/WC), but retained almost wild-type ratios of released (Sup) to cell-associated (Cell) amounts of the minor components (Fig. 3, compare lanes ORF1 and WT in panels FimC/Cell through FimE/Sup). In the fim $C$ mutant, not only FimC itself but also FimD and FimE were absent (Fig. 3, lanes fimC in panels FimC/WC through FimE/ WC). Similarly, the fimD mutant did not have FimE or FimD itself (Fig. 3, lanes fimD in panels FimC/WC through FimE/WC). Thus, the inactivation of the fimC or fimD gene resulted in concomitant loss of the downstream gene products, probably due to polar effects of the insertions. The inactivation of fimE did not have a significant effect on the levels of FimC but had a slight negative effect on the level of FimD (Fig. 3, compare lanes fimE and WT in panels FimC/WC and FimD/WC). The fimD and fimE mutations were also shown to affect cellular localization of the remaining minor components; FimC in the fimD mutant and FimC and FimD in the fimE mutant appeared to leak out into the culture supernatant (Fig. 3, lane fimD in panel FimC/Sup and lanes fimE in panels FimC/Sup and FimD/ Sup). Consistent with this finding, no minor components were found in fimbriae purified from the fim $C$, fimD and fimE mutants (Fig. 2, lane fimC, fimD and fimE). These results suggest that FimE is required for the assembly of FimC and FimD onto a fimbrial fibre consisting of fimbrilin (FimA). The strong band immediately below the FimC band (Fig. 3 FimC panels WC and Cell) is an unrelated protein cross-reacting with anti-FimC antibody.

Next, we examined the cellular amount of FimA in the same TCA-precipitated samples by immunoblotting with anti-FimA antibody (Fig. 3, panels FimA). Quantitative image analyses using the software Image J (http:// rsb.info.nih.gov/ij/) showed that the FimA signals in whole cultures of the ORF1, fim C, fimD and fimE mutants were approximately $80 \%$ of that of wild-type (Fig. 3, panel FimA/WC). Interestingly, immunoblotting with pellets (Fig. 3, panel FimA/Cell) and culture supernatants (Fig. 3, panel FimA/Sup) gave different profiles for the mutants. The FimA signals in the pellets from the fim $C$, fimD and fimE mutants were approximately $50 \%$ of that of wildtype, whereas the signal of the ORF1 mutant was approximately $80 \%$. The FimA signals in the culture supernatants of the fimC, fimD and fimE mutants were about twice that of the wild-type. After ultracentrifugation of the culture supernatants, FimA was detected in the precipitates, suggesting that it was released as filaments rather than monomers (data not shown). Thus, fimbriae of the fim $C$, fimD and fimE mutants seem more apt to detach from cells than those of the wild-type. Since all of the fim $C$, 
fimD and fimE mutants lack FimE (Fig. 3, panel FimE/ WC), it appears that the loss of FimE destabilizes the fimbrial attachment to the cell surface.

\section{Autoaggregation activities of the ORF1, fimC, fimD and fimE mutants}

We then examined whether the minor components are involved in autoaggregation of $P$. gingivalis, which seems to depend on the formation of the FimA fimbriae (Tokuda et al., 1996) and to have some correlation with colonization (Tokuda et al., 1996) and virulence (Eick et al., 2002). Wild-type and mutant cells, gently washed with PBS, were prepared, and each suspension $(2 \mathrm{ml})$ was shaken in a test tube. Formation of aggregates and cleared supernatants of wild-type cells were apparent just by eye, whereas the fimC, fimD and fimE mutants, but not the ORF1 mutant, showed much less aggregation (Fig. 4a). For quantitative analysis, the $\mathrm{OD}_{660}$ value of each suspension was monitored

(a)

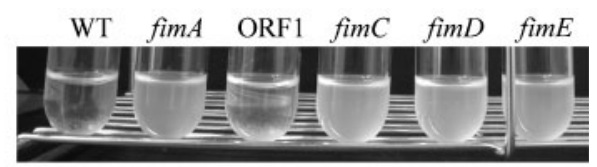

(b)

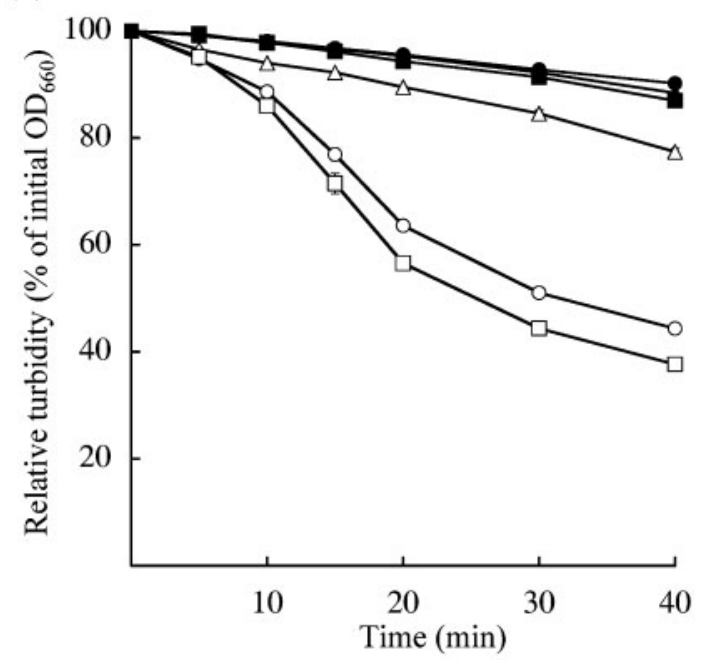

Fig. 4. Autoaggregation activities of the wild-type and mutant $P$. gingivalis strains. (a) Aggregation of cells after incubation for $60 \mathrm{~min}$. Strains are labelled as described in the legend to Fig. 3. (b) Time-course of autoaggregation. The $\mathrm{OD}_{660}$ value of each cell suspension was measured at the indicated times. Relative turbidities, defined as the relative $\mathrm{OD}_{660}$ values (in percent) normalized to the initial value of each suspension (i.e. at time zero; approximately 0.7 ), are plotted against incubation time. All assays were performed in triplicate and the means \pm SE are shown (standard errors not shown where smaller than symbols). $\bigcirc$, Wild-type strain ATCC 33277; $\triangle$, fimA mutant KDP98; $\square$, ORF1 mutant KO1; •, fimC mutant OZ5001C; $\mathbf{\Delta}$, fimD mutant KO3;, fimE mutant KO4.
(Fig. 4b). The $\mathrm{OD}_{660}$ values for the wild-type and the ORF1 mutant strains decreased gradually during constant shaking, reaching $44 \%$ and $38 \%$ of the initial $\mathrm{OD}_{660}$ values, respectively, after $40 \mathrm{~min}$, indicative of autoaggregation activity. In contrast, the fim $C$, fimD and fimE mutants still had relative $\mathrm{OD}_{660}$ values of approximately $86-90 \%$, which were actually slightly higher than that of the fimA mutant $(77 \%)$. Continuous shaking could detach fimbriae from cells during the autoaggregation experiment, although diluted cell suspensions were shaken gently. In fact, the fim C, fimD and fimE mutants seemed to release their fimbriae into the medium during growth (Fig. 3, panel FimA/Sup). However, immunoblotting assays detected no significant decrease in the amount of bound fimbriae in any of the mutant strains after 40 min shaking (data not shown). Therefore, we conclude that the attenuated autoaggregation activities of the fimC, fimD and fimE mutants resulted from the lack of FimE or other minor component(s) of the fimbriae rather than the loss of fimbriae themselves.

\section{Binding of fimbriae purified from the wild-type, fimC, fimD and fimE strains to rGAPDH and extracellular matrix proteins}

We also examined the binding activities of purified fimbriae to rGAPDH from Streptococcus oralis and the two extracellular matrix proteins, fibronectin and type I collagen, which have been reported by several groups (Maeda et al., 2004a; Nakamura et al., 1999). The ELISA results demonstrated that fimbriae from the wild-type bind to these proteins, whereas those from the fim $C$, fimD and fimE mutants showed significantly lower binding (Fig. 5). These binding experiments were based on the assumption that the wild-type and mutant fimbrial preparations (i.e. fimbriae with and without minor components) have similar reactivity with the anti-fimbriae serum. Indeed, control experiments using wells coated only with fimbriae showed no significant difference in reactivity among the mutant fimbriae. Since all three mutants lack FimE (Fig. 3, panel FimE/WC, lanes fimC, fimD and fimE) and the fimE fimbriae lack FimC and FimD (Fig. 2, panels Anti-FimC and Anti-FimD), these results suggest that some of the three minor components are involved in the binding of the FimA fimbriae to these bacterial and eukaryotic proteins. Which minor component(s) serve directly as adhesin(s) remains to be elucidated.

\section{DISCUSSION}

In this study, we characterized the four genes (ORF1, fimC, fimD and fimE) downstream of fimA, the gene for a major subunit of long fimbriae (Fig. 1) and found that the most distal gene fimE encodes a minor component that is closely associated with the intact FimA fimbriae (Fig. 2) as previously reported for the other two genes fim $C$ and fimD (Yoshimura et al., 1993). Fimbriae isolated from the fimC, 

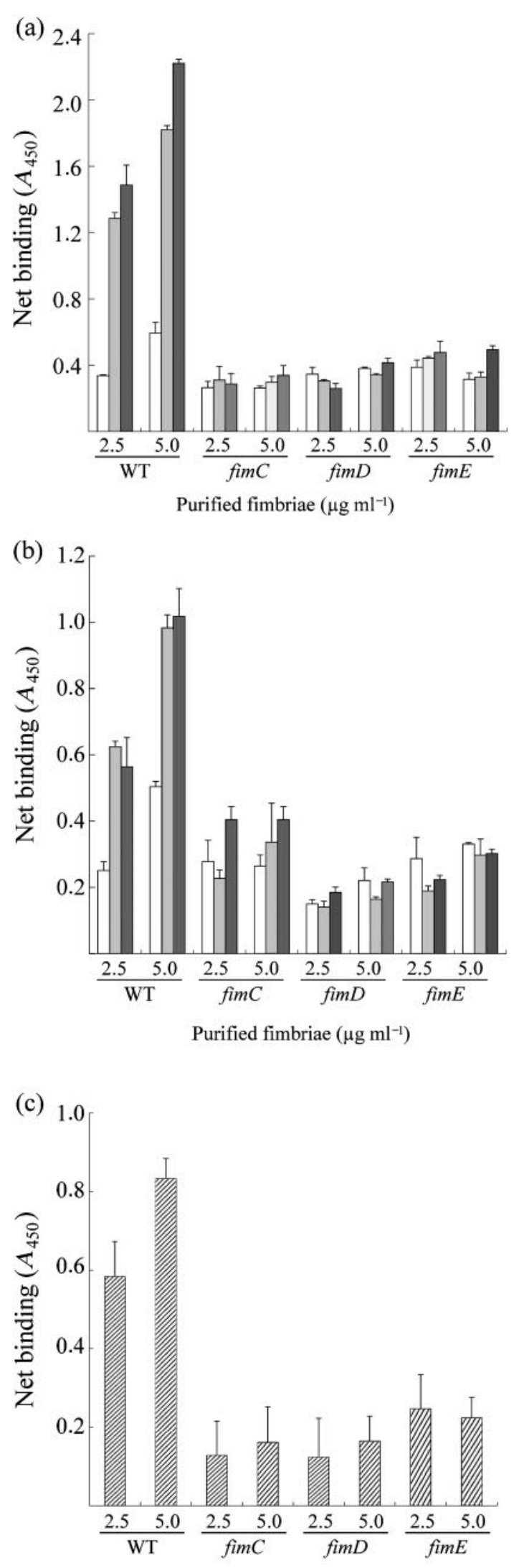

Purified fimbriae $\left(\mu \mathrm{g} \mathrm{ml}^{-1}\right)$
Fig. 5. Specific binding of the wild-type and mutant fimbriae to prokaryotic and eukaryotic proteins. $(a, b)$ Binding of fimbriae to rGAPDH (a) and fibronectin (b). Wells of microtitre plates were treated with buffer containing casein but without specific ligands (white bars), or with $10 \mu \mathrm{g} \mathrm{ml}^{-1}$ (light grey bars) or $20 \mu \mathrm{g} \mathrm{ml}^{-1}$ (dark grey bars) rGAPDH (a) or fibronectin (b) and then with fimbriae $\left(2.5\right.$ or $5.0 \mathrm{mg} \mathrm{ml}^{-1}$ ) purified from the wild-type, fimC, fimD or fimE strains (for detailed procedures see Methods). (c) Binding of fimbriae to type I collagen. Wells of microtitre plates pre-coated with type I collagen were treated with purified fimbriae as described above. For each sample, the mean $A_{450}$ value of the control reactions was subtracted from that with fimbriae and the resulting value was defined as net binding. Bars indicate the means \pm SE values of each combination (in triplicate).

fimD and fimE mutants had lost all the three minor components (Fig. 2), although FimC and FimD were produced and detected in the fimE mutant (Fig. 3). Fimbriae from all of these mutants had lost autoaggregation ability (Fig. 4) and the ability to adhere to the bacterial and eukaryotic ligands, GAPDH, fibronectin and collagen type I (Fig. 5). Since all of these mutants lack FimE, our results suggest that FimE at least is involved in the adhesive activities of the FimA fimbriae. Incorporation of minor components into mature fimbriae and their contribution to the adhesive function are commonly found in various bacterial pili as reported for type I fimbriae and P pili of $E$. coli (Jones et al., 1995; Kuehn et al., 1992).

It is unclear whether FimE is directly involved in adhesion functions. In the fimE mutant, FimC and FimD were produced and detected in whole cultures (Fig. 3), but they were not incorporated into fimbriae (Fig. 2) and were released into the culture supernatant (Fig. 3), suggesting that FimE is required for the integration of FimC and FimD into the mature and functional fimbriae. FimE could be required for the assembly of a putative tip complex consisting of FimC, FimD and FimE, one or more of which might serve as adhesin(s). Alternatively, FimE might not be directly responsible for adhesion but could help adhesin(s) FimC and/or FimD incorporate into fimbriae, e.g. by serving as a molecular chaperone. Preliminary analyses of the FimC, FimD and FimE sequences failed to detect a significant level of similarity to any of the known adhesins or other fimbrial proteins. It will be necessary to test whether any of these minor components can bind directly to the bacterial and eukaryotic ligands.

The fimC mutant simultaneously lost the ability to produce FimD and FimE, and the fimD mutant lacks FimE. A simple interpretation of these polar effects is that the genes from fimC through fimE may form an operon and that their translation is coupled. Translational coupling is a commonly used strategy to maintain strict stoichiometry of components of supramolecular complexes, including fimbriae (Draper, 1996). Similarly, the fimA mutant lacks all of the minor components (Fig. 3). However, this cannot be attributed to a polar effect of the insertion since the 
fimA mRNA has been reported to be monocistronic (Amano et al., 1994; Dickinson et al., 1988) and the replacement of ORF1, the gene immediately downstream of fimA and immediately upstream of fimC (Fig. 1), by the $\mathrm{Em}^{\mathrm{r}}$ cassette did not significantly affect the amounts of the minor components (Fig. 3). There might be some regulatory circuit to prevent synthesis of the minor components in the absence of fimbriae. Also, it appears that the level of the fimD product is decreased in the fimE mutant (Fig. 3, panel FimD/WC). The mechanism of this negative effect of the fimE insertion on the upstream gene remains to be elucidated.

Among the four genes downstream of fimA, ORF1 appears to play a minor role in fimbriae assembly and function since its inactivation gave a very weak or no phenotype. The ORF1 sequences of the fimA genotype I strains 381 and ATCC 33277 were identical (357 bp in length) and shorter than the corresponding gene PG2133 (909 bp in length) of W83, a poorly fimbriated strain. The $15 \mathrm{kDa}$ ORF1 and $30 \mathrm{kDa}$ PG2133 products were not detected in whole cell samples or in purified fimbriae (data not shown). The ORF1 mutant showed a slight decrease in FimA, but the ratio of cell-associated/culture supernatant FimA and the autoaggregation activity were very close to those of wild-type (Fig. 3). Taking these results together, ORF1 is not likely to be involved in fimbrial formation at least in strains ATCC 33277 and 381. However, the ORF1 mutant showed a slight but reproducible increase in autoaggregation (Fig. 4). Therefore, the ORF1 product might play a role in the negative regulation of adhesion, although its contribution appears to be marginal, at least under the conditions tested.

Fimbriae from strains other than those carrying fimA of genotype I have not been purified thus far, and their morphology and antigenic variations have not been explored. Our preliminary purification data suggest that fimbriae from several fimA genotype Ib (close to genotype I) and II strains have at least three minor components equivalent to those from the genotype I strains 381 and ATCC 33277 but do not contain a component corresponding to the gene product of PG2133 (or ORF1) (S. N. \& F. Y., unpublished results). It will be of great interest in terms of molecular function and evolution to compare the sequences and the natures of the minor components of the FimA fimbriae with different fimA genotypes, especially those of genotype II, which is predominant among periodontitis patients (Amano et al., 2000).

\section{ACKNOWLEDGEMENTS}

We thank Dr Michio Homma (Nagoya University) for invaluable discussions and encouragement. We also thank the Institute for Genomic Research (TIGR) for making the P. gingivalis W83 genomic sequence freely available to the public. This work was supported by Grants-in-Aid for Scientific Research (17791318 to S. N. and 15591957 to F. Y.) from the Japan Society for the Promotion of Science (JSPS) and the AGU High-Tech Research Center Project from
The Ministry of Education, Culture, Sports, Science and Technology, Japan.

\section{REFERENCES}

Amano, A., Sharma, A., Sojar, H. T., Kuramitsu, H. K. \& Genco, R. J. (1994). Effects of temperature stress on expression of fimbriae and superoxide dismutase by Porphyromonas gingivalis. Infect Immun 62 , 4682-4685.

Amano, A., Kataoka, K., Raj, P. A., Genco, R. J. \& Shizukuishi, S. (1996). Binding sites of salivary statherin for Porphyromonas gingivalis recombinant fimbrillin. Infect Immun 64, 4249-4254.

Amano, A., Fujiwara, T., Nagata, H., Kuboniwa, M., Sharma, A., Sojar, H. T., Genco, R. J., Hamada, S. \& Shizukuishi, S. (1997). Porphyromonas gingivalis fimbriae mediate coaggregation with Streptococcus oralis through specific domains. J Dent Res 76, 852-857.

Amano, A., Nakamura, T., Kimura, S., Morisaki, I., Nakagawa, I., Kawabata, S. \& Hamada, S. (1999). Molecular interactions of Porphyromonas gingivalis fimbriae with host proteins: kinetic analyses based on surface plasmon resonance. Infect Immun 67, 2399-2405.

Amano, A., Kuboniwa, M., Nakagawa, I., Akiyama, S., Morisaki, I. \& Hamada, S. (2000). Prevalence of specific genotypes of Porphyromonas gingivalis fimA and periodontal health status. J Dent Res 79, 1664-1668.

Amano, A., Nakagawa, I., Okahashi, N. \& Hamada, N. (2004). Variations of Porphyromonas gingivalis fimbriae in relation to microbial pathogenesis. J Periodontal Res 39, 136-142.

Andrian, E., Grenier, D. \& Rouabhia, M. (2006). Porphyromonas gingivalis-epithelial cell interactions in periodontitis. J Dent Res 85, 392-403.

Dickinson, D. P., Kubiniec, M. A., Yoshimura, F. \& Genco, R. J. (1988). Molecular cloning and sequencing of the gene encoding the fimbrial subunit protein of Bacteroides gingivalis. J Bacteriol 170, $1658-1665$.

Draper, D. (1996). Translational initiation. In Escherichia coli and Salmonella, pp. 902-908. Edited by R. C. I. F. C. Neidhart, J. L. Ingraham, E. C. C. Lin, K. B. Low, B. Magasanik, W. S. Reznikoff, M. Riley, M. Schaecheter \& H. E. Umbarger. Washington, DC: American Society for Microbiology.

Eick, S., Rodel, J., Einax, J. W. \& Pfister, W. (2002). Interaction of Porphyromonas gingivalis with KB cells: comparison of different clinical isolates. Oral Microbiol Immunol 17, 201-208.

Fletcher, H. M., Schenkein, H. A., Morgan, R. M., Bailey, K. A., Berry, C. R. \& Macrina, F. L. (1995). Virulence of a Porphyromonas gingivalis W83 mutant defective in the prtH gene. Infect Immun 63, 1521-1528.

Hajishengallis, G., Tapping, R. I., Harokopakis, E., Nishiyama, S., Ratti, P., Schifferle, R. E., Lyle, E. A., Triantafilou, M., Triantafilou, K. \& Yoshimura, F. (2006). Differential interactions of fimbriae and lipopolysaccharide from Porphyromonas gingivalis with the Toll-like receptor 2-centered pattern recognition apparatus. Cell Microbiol 8, 1557-1570.

Hamada, S., Amano, A., Kimura, S., Nakagawa, I., Kawabata, S. \& Morisaki, I. (1998). The importance of fimbriae in the virulence and ecology of some oral bacteria. Oral Microbiol Immunol 13, 129-138.

Hasegawa, Y., Nishiyama, S., Nishikawa, K., Kadowaki, T., Yamamoto, K., Noguchi, T. \& Yoshimura, F. (2003). A novel type of two-component regulatory system affecting gingipains in Porphyromonas gingivalis. Microbiol Immunol 47, 849-858.

Hongo, H., Osano, E., Ozeki, M., Onoe, T., Watanabe, K., Honda, O., Tani, H., Nakamura, H. \& Yoshimura, F. (1999). Characterization of 
an outer membrane protein gene, pgmA, and its gene product from Porphyromonas gingivalis. Microbiol Immunol 43, 937-946.

Jones, C. H., Pinkner, J. S., Roth, R., Heuser, J., Nicholes, A. V., Abraham, S. N. \& Hultgren, S. J. (1995). FimH adhesin of type I pili is assembled into a fibrillar tip structure in the Enterobacteriaceae. Proc Natl Acad Sci U S A 92, 2081-2085.

Kontani, M., Ono, H., Shibata, H., Okamura, Y., Tanaka, T., Fujiwara, T., Kimura, S. \& Hamada, S. (1996). Cysteine protease of Porphyromonas gingivalis 381 enhances binding of fimbriae to cultured human fibroblasts and matrix proteins. Infect Immun 64, 756-762.

Kuehn, M. J., Heuser, J., Normark, S. \& Hultgren, S. J. (1992). P pili in uropathogenic E. coli are composite fibres with distinct fibrillar adhesive tips. Nature 356, 252-255.

Lamont, R. J. \& Jenkinson, H. F. (1998). Life below the gum line: pathogenic mechanisms of Porphyromonas gingivalis. Microbiol Mol Biol Rev 62, 1244-1263.

Lamont, R. J. \& Jenkinson, H. F. (2000). Subgingival colonization by Porphyromonas gingivalis. Oral Microbiol Immunol 15, 341-349.

Lugtenberg, B., Meijers, J., Peters, R., van der Hoek, P. \& van Alphen, L. (1975). Electrophoretic resolution of the 'major outer membrane protein' of Escherichia coli K12 into four bands. FEBS Lett 58, 254-258.

Maeda, K., Nagata, H., Kuboniwa, M., Kataoka, K., Nishida, N., Tanaka, M. \& Shizukuishi, S. (2004a). Characterization of binding of Streptococcus oralis glyceraldehyde-3-phosphate dehydrogenase to Porphyromonas gingivalis major fimbriae. Infect Immun 72, 5475-5477.

Maeda, K., Nagata, H., Yamamoto, Y., Tanaka, M., Tanaka, J., Minamino, N. \& Shizukuishi, S. (2004b). Glyceraldehyde-3-phosphate dehydrogenase of Streptococcus oralis functions as a coadhesin for Porphyromonas gingivalis major fimbriae. Infect Immun 72, 1341-1348.

Nagano, K., Read, E. K., Murakami, Y., Masuda, T., Noguchi, T. \& Yoshimura, F. (2005). Trimeric structure of major outer membrane proteins homologous to OmpA in Porphyromonas gingivalis. $J$ Bacteriol 187, 902-911.

Nakamura, T., Amano, A., Nakagawa, I. \& Hamada, S. (1999). Specific interactions between Porphyromonas gingivalis fimbriae and human extracellular matrix proteins. FEMS Microbiol Lett 175, 267-272.

Nelson, K. E., Fleischmann, R. D., DeBoy, R. T., Paulsen, I. T., Fouts, D. E., Eisen, J. A., Daugherty, S. C., Dodson, R. J., Durkin, A. S. \& other authors (2003). Complete genome sequence of the oral pathogenic bacterium Porphyromonas gingivalis strain W83. J Bacteriol 185, 5591-5601.

Nishikawa, K., Yoshimura, F. \& Duncan, M. J. (2004). A regulation cascade controls expression of Porphyromonas gingivalis fimbriae via the FimR response regulator. Mol Microbiol 54, 546-560.
Park, Y., Simionato, M. R., Sekiya, K., Murakami, Y., James, D., Chen, W., Hackett, M., Yoshimura, F., Demuth, D. R. \& Lamont, R. J. (2005). Short fimbriae of Porphyromonas gingivalis and their role in coadhesion with Streptococcus gordonii. Infect Immun 73, 3983-3989.

Smith, K. A. \& Salyers, A. A. (1989). Cell-associated pullulanase from Bacteroides thetaiotaomicron: cloning, characterization, and insertional mutagenesis to determine role in pullulan utilization. $J$ Bacteriol 171, 2116-2123.

Soto, G. E. \& Hultgren, S. J. (1999). Bacterial adhesins: common themes and variations in architecture and assembly. J Bacteriol 181, 1059-1071.

Takahashi, Y., Yoshimura, F., Kawanami, M. \& Kato, H. (1992). Detection of fimbrilin gene (fimA) in Porphyromonas (Bacteroides) gingivalis by Southern blot analysis. J Periodontal Res 27, 599-603.

Takahashi, Y., Yoshimoto, H., Kato, D., Hamada, N., Arai, M. \& Umemoto, T. (2001). Reduced fimbria-associated activities of Porphyromonas gingivalis induced by recombinant fimbrial expression. FEMS Microbiol Lett 195, 217-222.

Tokuda, M., Duncan, M., Cho, M. I. \& Kuramitsu, H. K. (1996). Role of Porphyromonas gingivalis protease activity in colonization of oral surfaces. Infect Immun 64, 4067-4073.

Watanabe, K., Onoe, T., Ozeki, M., Shimizu, Y., Sakayori, T., Nakamura, H. \& Yoshimura, F. (1996). Sequence and product analyses of the four genes downstream from the fimbrilin gene ( fimA) of the oral anaerobe Porphyromonas gingivalis. Microbiol Immunol 40, 725-734.

Watanabe-Kato, T., Hayashi, J. I., Terazawa, Y., Hoover, C. I., Nakayama, K., Hibi, E., Kawakami, N., Ikeda, T., Nakamura, H. \& other authors (1998). Isolation and characterization of transposoninduced mutants of Porphyromonas gingivalis deficient in fimbriation. Microb Pathog 24, 25-35.

Yoshimura, F., Takahashi, K., Nodasaka, Y. \& Suzuki, T. (1984). Purification and characterization of a novel type of fimbriae from the oral anaerobe Bacteroides gingivalis. J Bacteriol 160, 949-957.

Yoshimura, F., Watanabe, K., Takasawa, T., Kawanami, M. \& Kato, H. (1989). Purification and properties of a 75-kilodalton major protein, an immunodominant surface antigen, from the oral anaerobe Bacteroides gingivalis. Infect Immun 57, 3646-3652.

Yoshimura, F., Takahashi, Y., Hibi, E., Takasawa, T., Kato, H. \& Dickinson, D. P. (1993). Proteins with molecular masses of 50 and 80 kilodaltons encoded by genes downstream from the fimbrilin gene ( $\operatorname{imA}$ ) are components associated with fimbriae in the oral anaerobe Porphyromonas gingivalis. Infect Immun 61, 5181-5189.

Edited by: P. E. Kolenbrander 